\title{
El hábito de tallar el paisaje verde. Tecnología lítica en el sector septentrional de El Alto - Ancasti (Catamarca)
}

\section{(1) Ignacio Gerola*}

\author{
Fecha de defensa: 26 de octubre de 2018 \\ Directora: Dra. Inés Gordillo \\ Co-director: Dr. José María Vaquer \\ Jurados: Dres. Juan P. Carbonelli y Federico \\ Restifo
}

La presente tesis consiste en el estudio de la organización de la tecnología lítica de los habitantes de tres sitios del Formativo (primer milenio A.D.) del sector septentrional de El Alto Ancasti. El abordaje de la organización lítica de estas poblaciones otorga la posibilidad de avanzar hacia la caracterización e interpretación de las ocupaciones, de las prácticas sociales vinculadas a la producción lítica y del aprovechamiento de los recursos locales.

\section{Área de estudio}

La Sierra El Alto Ancasti pertenece al sistema de Sierras Pampeanas Noroccidentales. Se ubica en la franja sudoriental de la Provincia de Catamarca, en posición intermedia entre los valles occidentales de Catamarca y las Tierras Bajas del límite provincial Este. Este sistema serrano es un cordón ininterrumpido, uniforme, cuyas alturas máximas rondan los $2.000 \mathrm{~m}$ s.n.m. Presenta tres pisos ambientales: pastizales de altura, arbustal-pastizal y bosque serrano.

\section{Antecedentes}

Tradicionalmente se concebía a El Alto Ancasti como un espacio de transición, de obtención de recursos específicos y como un sector periférico de los centros de desarrollo ubicados en los valles occidentales (Ardissone, 1945; González, 1964, 1979; Pérez Gollán y Heredia, 1987). En contraste, los estudios desarrollados durante la última década y media postulan la presencia de ocupaciones permanentes, de larga data, con importantes inversiones en infraestructura agrícola y habitacional, el empleo de estrategias y tecnologías adecuadas en la explotación de los recursos locales; un vínculo cotidiano entre los aleros y cuevas con arte rupestre, los espacios de vivienda y los de producción (pastoril, agrícola y tecnológica); una diacronía considerable en las producciones rupestres, que exceden iconográfica y temporalmente el vínculo con Aguada; y una

\footnotetext{
* Instituto de Arqueología, Facultad de Filosofía y Letras, Universidad de Buenos Aires (UBA). 25 de mayo 217, 3ㅇp piso (CP C1002ABE) Ciudad Autónoma de Buenos Aires, Argentina. E-mail: ignaciogerola@gmail.com
}

economía campesina autosuficiente en conjuntos aldeanos estables (Egea, 2015; Eguia, Prieto y Gerola, 2016; Gheco, 2012; Gordillo, Zuccarelli y Eguia, 2017; Moreno 2014; Quesada, Gastaldi y Granizo, 2012; Zuccarelli 2014).

\section{Objetivo e Hipótesis}

El objetivo general consiste en caracterizar la organización de la tecnología lítica, y las prácticas sociales asociadas a ella, de las ocupaciones acontecidas en distintos momentos del Formativo (primer milenio A.D.) en el sector septentrional de El Alto Ancasti.

Como hipótesis de trabajo se afirma que el uso exclusivo de materias primas locales y el empleo de estrategias expeditivas caracterizan la organización de la tecnología lítica de El Alto Ancasti septentrional, en las ocupaciones acontecidas durante el Formativo. Esto se debe a los requerimientos productivos de las actividades cotidianas, que implican el empleo de instrumentos de uso inmediato y generalizado, y a la autonomía de la producción lítica en el área.

\section{Sitios analizados}

El sitio Guayamba 2 se localiza en la localidad de Guayamba (departamento de El Alto). El sitio se localiza a $1.078 \mathrm{~m}$ s.n.m. y está conformado por 4 recintos. Dos estructuras rectangulares adosadas y a aproximadamente unos 50 metros en dirección noroeste, se localizan la estructura semicirular 3 y adosada a ella la estructura subrectangular 4 (Eguia et. al., 2016). Se obtuvieron dos fechados para las ocupaciones del sitio: $1050 \pm 30$ AP (Cal 950-1030 A.D.); y $1450 \pm 30$ AP (Cal 560-650 A.D.), ambos realizados sobre óseo de camélido. Se destaca el vínculo de Guayamba 2 con Casa Pintada, ubicada a una distancia de 190 metros en dirección sur (Gordillo et al., 2017).

La cueva Casa Pintada constituye un afloramiento rocoso metamórfico. Se encuentra en el ambiente de bosque serrano a 1.079 m s.n.m. En el centro del sector oeste aflora una roca que posee un mortero y en el sector noreste de la cueva, por fuera de la línea de goteo, posee una superficie nivelada mediante muro. El sitio cuenta con 14 motivos rupestres. La cueva ha sido adscripta al período Medio, en base a la presencia de representaciones de huellas de felino, que suelen ser asociadas a Aguada, no obstante, esta asociación no se considera suficiente y se requiere la contrastación de nuevas evidencias (Eguia et al., 2016). 
Rodeo de los Indios (o Rodeo 3 ) se define como un conjunto habitacional localizado en el sector cumbral de los pastizales de altura, compuesto por recintos habitacionales y áreas de cultivo. Está conformado por dieciocho recintos de piedra cuadrangulares. Se organizan en dos sectores o núcleos de estructuras adosadas, ambos ubicados en la misma lomada (Zuccarelli, 2014). Fechado en $1.464 \pm 36$ AP (carbón vegetal; $\delta{ }^{13} \mathrm{C}=-24,3 \%$ ) y $1.305 \pm 36 \mathrm{AP}$ (carbón vegetal; $\delta{ }^{13} \mathrm{C}=-24,3 \%$ ). El calibrado de estas edades ubica la ocupación del sitio entre ca. 570-770 A.D.

\section{Marco Teórico}

El abordaje teórico posee como fin llevar a cabo una discusión sobre las concepciones que se poseen sobre "el tiempo y el espacio" al abordar un caso de estudio. Se retoman las discusiones de tiempo y temporalidad esbozadas por Alfred Gell (1992) y Gavin Lucas (2005). Y se destaca la "Perspectiva de Habitar" de Ingold (2000) que guía la matriz teórica de este trabajo.

\section{Metodología}

En primer lugar, se diferencian las materias primas registradas en los sitios. Esto se pone en relación con las fuentes de materias primas primarias y secundarias relevadas en las prospecciones (Nami, 1992).

En segundo orden, se realiza la caracterización técnico morfológica y morfológica funcional de los conjuntos a nivel macroscópico, para la cual se emplea terminología morfológica descriptiva elaborada por Aschero $(1975,1983)$. Se inicia con la segmentación de cada conjunto, partiendo del criterio de clase tipológica y luego se aplican los criterios de clase técnica y serie técnica (Aschero y Hocsman, 2004) que permiten distinguir los grados de trabajo invertidos en la producción de instrumentos y los diversos procedimientos técnicos ejecutados en la producción.

Además, se evalúa la relevancia que tuvo cada materia prima en la elaboración de cada grupo tipológico, con el fin de inferir si existe una selección diferenciada acorde al tipo de instrumento a confeccionar.

El desarrollo de estos pasos metodológicos habilita a la comprensión del tipo de estrategias empleadas en la organización de la tecnología lítica (Nelson, 1991) en cada uno de los sitios estudiados.

\section{Resultados}

En los conjuntos de los tres sitios analizados, Guayamba 2 (G2), Casa Pintada (CP) y Rodeo de los Indios (R3), podemos observar un claro predominio del aprovechamiento del cuarzo como materia prima, en diversas variedades que van desde cuarzo blanco con brillo vítreo hasta cuarzo translúcido. Este recurso se presenta de forma abundante en las inmediaciones de los tres sitios, tanto en fuentes primarias como secundarias. La confección de unos pocos instrumentos en distintas variedades de Sílice en Guayamba 2 y en Casa Pintada constituye la única excepción al uso del cuarzo.

Se ha empleado talla directa y talla bipolar en E1 y E3 de $\mathrm{G}_{2}$, en CP y en Rodeo 3. No se ha podido encontrar aún evidencia de talla bipolar en E2 de G2. En tanto, en la E3 de $\mathrm{G} 2$ se realizaron desde las primeras etapas hasta las instancias finales de la producción. En cambio, en CP se habrían desarrollado solo las instancias avanzadas, como pueden ser la reducción de formas base y la formatización de filos y el mantenimiento. El material de las estructuras 1 y 2 de $G_{2}$ y del sitio $R_{3}$ no nos permite inferir etapas productivas específicas.

En cuanto a los instrumentos, los tres sitios exhiben una manufactura simple y expeditiva, en la que prevalece el microretoque marginal unifacial aprovechando biseles aptos para la regularización de filos.

Los cinco conjuntos analizados presentan una considerable diversidad instrumental. Los grupos tipológicos raspador, raedera, cuchillo y artefacto de retoques sumarios se encontraron en todos ellos. Se han recuperado núcleos en $G_{2}$ y $R_{3}$, todos de cuarzo y en su mayoría designados en la categoría lascados aislados. No se han encontrado núcleos en CP.

En los tres sitios abordados se ha identificado el empleo de las técnicas de talla directa y bipolar. Debido a la dureza del cuarzo, la talla bipolar permite la obtención de formas base de menor espesor y de bisel más agudo. Más adecuadas para confeccionar ciertos tipos de instrumentos (por ejemplo cortantes), que aquellas obtenidos por talla directa, que suelen ser de mayor espesor y bisel más abrupto (Egea, 2015; Moreno, 2014).

A su vez, no se registra un aprovechamiento diferencial de las materias primas. Todas las categorías de instrumentos han sido elaboradas en diferentes variedades de cuarzo, en proporción semejante a como se presenta la variedad de este recurso en el terreno circundante a cada asentamiento.

\section{Discusión y Conclusiones}

En los sitios Guayamba 2, Casa Pintada y Rodeo 3 se ha aprovechado de forma casi exclusiva el cuarzo en sus diferentes variedades. Esta materia prima se encuentra de forma abundante en el paisaje inmediato a los tres sitios. En este sentido, al ser una roca muy dura, el cuarzo otorga la ventaja de que una vez que se ha obtenido un 
filo apto, este suele ofrecer buena resistencia y durabilidad (Moreno, 2014). Por lo tanto, habría sido una materia prima suficiente para la producción de una variedad importante de instrumentos, acorde a los requerimientos de diversas actividades cotidianas. Cabe destacar que habría sido posible el aprovechamiento de materias primas de mejor calidad para la talla, pero esto implicaría el acceso a otras áreas o el intercambio con grupos no locales. Se considera que la selección del cuarzo en Guayamba 2, Casa Pintada y Rodeo 3, implica un compromiso con el paisaje local (Moreno, 2014), relacionado con ocupaciones permanentes y autosuficientes. Se entiende que los habitantes poseerían una relación de larga data con el paisaje circundante que permitió el desarrollo del conocimiento acabado de sus recursos y el perfeccionamiento de las técnicas adecuadas para explotarlos.

En cuanto a los instrumentos, los tres sitios registran una manufactura simple y de baja inversión de trabajo, que aprovecharía cualquier bisel apto para formatizar filos. Este tipo de manufactura sería suficiente para producir una buena variedad de instrumentos. Diversidad artefactual que habría permitido realizar diferentes acciones físicas sobre distintos tipos de materiales (Egea, 2015). Es decir, instrumentos capaces de satisfacer las demandas de diversos tipos de actividades cotidianas (Moreno, 2014).

Además, la estructura 3 de Guayamba 2 habría funcionado como un espacio donde se habrían desarrollado todas las etapas de la secuencia productiva, lo que refiere a un tipo de producción terminal (Ericson, 1984). En cambio, en Casa Pintada se desplegarían las etapas finales de una producción de carácter secuencial (Ericson, 1984). En tanto, las Estructuras 1 y 2 de Guayamba, y las estructuras 3 b y 6 de Rodeo 3, indican diversas prácticas de talla y un carácter proporcionalmente más vinculado al uso de los instrumentos que a su confección, en comparación con E3 de G2 y Casa Pintada. Las diferencias que percibimos en la producción lítica de los tres sitios analizados se deben más bien a las características propias de los asentamientos que a su ubicación ambiental y altitudinal o a su proximidad.

La hipótesis de trabajo ha sido corroborada, la evidencia apunta a una producción lítica autónoma e independiente, basada en el aprovechamiento de recursos locales, que fue eficiente a la hora de producir una variedad considerable de instrumentos que pudieran cumplir con los requerimientos de las diversas actividades cotidianas. Entonces, se afirma que la organización lítica de Guayamba 2, Casa Pintada y Rodeo 3, durante el período Formativo se caracteriza por el aprovechamiento de recursos líticos locales e inmediatos y el empleo de estrategias expeditivas en la producción lítica.
Todos los elementos de este paisaje humano prehispánico apuntan en dirección de las interpretaciones que enfatizan los desarrollos locales autónomos de El Alto-Ancasti, cuyas ocupaciones estarían caracterizadas por comunidades asentadas de forma permanente con economías diversificadas y autosuficientes.

\section{Referencias citadas}

»Aschero, C. (1975). Ensayo para una clasificación morfológica de artefactos líticos aplicada a estudios tipológicos comparativos. Informe presentado al CONICET. Manuscrito inédito.

» Aschero, C. (1983). Ensayo para una clasificación morfológica de artefactos líticos. Apéndice A y B. Cátedra de Ergología y Tecnología, Facultad de Filosofía y Letras, Universidad de Buenos Aires. Manuscrito inédito.

»Aschero, C. y Hocsman, S. (2004). Revisando cuestiones tipológicas en torno a la clasificación de artefactos bifaciales. En M. Ramos, A. Acosta y D. Loponte (eds.), Temas de Arqueología. Análisis Lítico (pp. 7-25). Lujan: Universidad Nacional de Lujan.

»Ardissone, R. (1945). Las Pircas de Ancasti. Contribución al conocimiento de los restos de andenes en el Noroeste Argentino. En F. De Aparicio, M.T. Grondona y E. Wernike (Eds.), GAEA Anales de la Sociedad Argentina de Estudios Geográficos 7 (pp. 383416). Buenos Aires: Sociedad Argentina de Estudios Geográficos.

»Egea, D. (2015). Tallando en espacios rupestres. Tecnología lítica en una cueva pintada del este catamarqueño. (Tesis de Licenciatura inédita), Universidad Nacional de Catamarca, Argentina.

»Eguia, L., Prieto, C. y Gerola, I. (2016). Guayamba 2: Abordando el espacio doméstico en los bosques orientales de Catamarca. Comechingonia, 20(2), 43-72.

»Ericson, J. E. (1984). Toward the analysis of lithic production systems. En J. Ericson y B. Purdy (Eds.), Prehistoric Quarries and Lithic Production (pp. 11-22). Cambridge: Cambridge University Press.

» Gell, A. (1992). The anthropology of time. Cultural constructions of temporal maps and images. Oxford: Berg.

» Gheco, L. (2012). Una Historia en la Pared. Hacia una Visión Diacrónica del Arte Rupestre de Oyola. (Tesis de Licenciatura inédita), Universidad Nacional de Catamarca, Argentina.

»González, A. R. (1964). La cultura de La Aguada del N.O. Argentino. Revista del Instituto de Arqueología, 3, $205-254$. 
" González, A. R. (1979). Dinámica cultural del Noroeste Argentino. Evolución histórica en las culturas del Noroeste Argentino. Antiquitas, 28-29, 1-15.

» Gordillo, I., Zuccarelli, V. y Eguia, L. (2017). Las casas del sol naciente. Arqueología de la vertiente oriental de El Alto Ancasti. En B. N. Ventura, G. Ortiz y M. B. Cremonte (Eds.), Arqueología de la vertiente oriental surandina. Interacción macro-regional, materialidades, economía y ritualidad (pp. 111-134). Buenos Aires: Sociedad Argentina de Antropología.

" Ingold, T. (2000). The Perception of the Enviroment. Essays on livehood, dwelling and skills. Londres: Routledge.

"Lucas, G. (2005). The Archaeology of Time. Londres: Routledge.

" Moreno, E. (2014). Materias primas, instrumentos líticos y prácticas domésticas en las sierras de El Alto-Ancasti, Catamarca. En: C. Mercuri y A. Elias (eds.), Cuadernos del Instituto Nacional de Antropología y Pensamiento Latinoamericano - Series especiales (pp. 141-16o).
"Nami, H. (1992). El subsistema tecnológico de la confección de instrumentos líticos y la explotación de los recursos del ambiente: Una nueva vía de aproximación. Shincal, 2, 33-53.

"Nelson, M. C. (1991). El estudio de la organización tecnológica. En M. B. Schiffer (Eds.), Archaeological Method and Theory 3 (pp. 57-100). Tucson: The University of Arizona Press.

"Pérez Gollán, J. A. y Heredia O. R. (1987). Hacia un replanteo de la cultura de La Aguada. Cuadernos Instituto Nacional de Antropología, 12, 161-178.

"Quesada, M., Gastaldi, M. y Granizo, G. (2012). Construcción de periferias y producción de lo local en las cumbres de El Alto-Ancasti. Relaciones de la Sociedad Argentina de Antropología, XXXVII(2), 435-456.

»Zuccarelli, V. (2014). Primeras aproximaciones al paisaje agrario del norte de la sierra El Alto Ancasti: un análisis multi-escalar. Arqueología, 20(1), 115-141. 